\title{
A novel automatic determination system for correction and simultaneous quantification of peroxidase and ascorbate in biosamples
}

\author{
Yong-Sheng $\mathrm{Li}^{1}$, Yang Zhao ${ }^{2}$, Qiao-Jing $\mathrm{LI}^{3}$, and Xiu-Feng Gao ${ }^{1}$ \\ ${ }^{1}$ Sichuan University \\ ${ }^{2}$ Sichuan University - Wangjiang Campus \\ ${ }^{3}$ Tokyo Daigaku
}

May 5, 2020

\begin{abstract}
Mastering synchronous changes of peroxidase (POD) activity concentration (EPOD) and ascorbic acid contents (cAsA) in biosamples can be used for estimation of ant-oxidative capability and healthy status of animals and plants, however, in determination of EPOD, the coexisted AsA produced serious interference. Therefore, to solve this problem and obtain true EPOD, this study deeply investigated the AsA interference using a guaiacol/POD/H2O2 reaction. Results manifest that instead of inactivation of the enzyme, the AsA causes the extinction of the chromogenic product formed in the reaction, resulting in the EPOD to deviate true value; this phenomenon occurs in all methods for quantification of EPOD. Because the interference of AsA is linear with the EPOD, the utilization of the linear correlation realized the correction of EPOD and the quantification of cAsA. Subsequently, the use of the extinction and coloration phenomena in the reaction, a new automatic analysis method and system were developed, and were used for the simultaneous determination of EPOD and cAsA in biosamples. After optimized, the variables of the system were obtained as follows: the determination ranges are 75 to $1300 \mathrm{U}^{*} \mathrm{~L}-1$ for EPOD and 5 to 100 mg* $\mathrm{L}-1$ for $\mathrm{cAsA}$; the detection limits are $22.5 \mathrm{U}^{*} \mathrm{~L}-1$ for EPOD and $1.67 \mathrm{mg}{ }^{*} \mathrm{~L}-1$ for cAsA; the relative standard deviation is less than $1.2 \%(\mathrm{n}=11)$; the sample throughput can reach 40 samples per hour; and the standard addition recovery is in the range of 95 to $105 \%$. This automatic method and system has been used successfully for accurate quantifications of EPOD and cAsA in various biosamples, and based on this, a new specialized analyzer can be also fabricated for the study of biosamples in biotechnology and bioengineering.
\end{abstract}

\section{Hosted file}

manuscript-lys.doc available at https://authorea.com/users/298350/articles/427563-anovel-automatic-determination-system-for-correction-and-simultaneous-quantificationof-peroxidase-and-ascorbate-in-biosamples

\section{Hosted file}

Figures-Lys.doc available at https://authorea.com/users/298350/articles/427563-a-novelautomatic-determination-system-for-correction-and-simultaneous-quantification-ofperoxidase-and-ascorbate-in-biosamples 